\title{
Was sind HTA - was bedeuten sie für den Arzt?
}

\author{
Michel Romanens ${ }^{a}$, \\ Franz Ackermann ${ }^{b}$, \\ Flavian Kurth \\ a Präsident Verein Ethik und \\ Medizin Schweiz (VEMS) \\ b Vorstand VEMS \\ c Sekretär VEMS
}

Ein Kommentar der FMH findet sich auf S. 1365.

Korrespondenz:

Dr. med. Michel Romanens Facharzt für Innere Medizin und Kardiologie Verein Ethik und Medizin Schweiz (VEMS) Ziegelfeldstrasse 1 $\mathrm{CH}-4600$ Olten

info[at]kardiolab.ch

www.vems.ch

\section{Rückblick DRG}

Wir erinnern uns: Vor gut einem Jahr wurden in der Schweiz die Fallpauschalen DRG eingeführt. Dieser Einführung vorausgegangen ist ein heftiger Widerstand, der sich in der «Aktion DRG Moratorium» formierte und, abgestützt auf eine starke Basis namhafter Persönlichkeiten des Gesundheitswesens als Mitunterzeichner, berechtigte Einwände gegen die diagnosebezogenen Fallkostenpauschalen ins Feld führte. Einwände, die noch heute im Raum stehen und auf www.drg-moratorium.ch im Detail nachgelesen werden können. Heute muss leider festgestellt werden, dass sie mindestens teilweise berechtigt waren und sind: Man wollte rationalisieren und sparen, jetzt haben wir bei höherem Verwaltungsaufwand, steigende Kosten und eine Tendenz zur Rationierung und zur Gefährdung der Patienten. Es ist klar, dass die Gründe hierfür komplexer sind, doch würde wohl kaum jemand die Einführung von DRG in der Schweiz heute als besonders geglückt bezeichnen.

\section{Verein Ethik und Medizin Schweiz (VEMS)}

Der Verein Ethik und Medizin Schweiz (VEMS) ist ein wissenschaftlicher Verein an der Schnittstelle von Medizin und Ethik. Er erhält sich dank der Finanzierung über die Fairfond Stiftung für Fairness im Gesundheitswesen ausschliesslich aus Donationen und Freiwilligenarbeit privater Individuen und Organisationen und ist unabhängig von sämtlichen Akteuren des Gesundheitswesens (Leistungserbringern, Krankenkassen, Industrie) sowie religionsneutral und nicht politisch. Der VEMS entwickelt seine Aktivitäten im Gesundheitswesen dort, wo aufgrund von Voreingenommenheiten und Verbindlichkeiten wissenschaftlich mangelhafte Grundlagen den Diskurs führen und in eine medizinisch-ethisch problematische Richtung zu lenken drohen. Dank seiner Unabhängigkeit kann der VEMS diese Grundlagen und ihre Implikationen unvoreingenommen hinterfragen und damit Impulse an andere, grössere Gremien und regulative Instanzen geben. Bei der Erarbeitung seiner Gutachten zieht der VEMS jeweils unabhängige Experten aus dem In- und Ausland bei. Das Verfassen der auf den Gutachten basierenden Stellungnahmen, Thesenpapiere und Vorschläge wird vom Sekretariat in Zusammenarbeit mit den Vorstandsmitgliedern geleistet, ebenso wie die Arbeit, diese zu kommunizieren. Ein schlankes Kernteam erlaubt so rasches Reagieren, die Verstärkung dieses Kernteams mit beigezogenen Spezialisten garantiert die fachliche Professionalität.

\section{Que sont les HTA et que signi- fient-ils pour le médecin?}

Le présent article passe en revue l'introduction de deux mesures, DRG et Managed care, destinées à réduire les coûts dans le secteur de la santé: alors que la première doit encore fournir la preuve de ses effets, la seconde a été rejetée par le peuple - d'autant plus qu'après mûres réflexions, la FMH ne pouvait plus la défendre. Par ailleurs, les auteurs relèvent que le Comité central de la FMH soutient les HTA sans les avoir expliqués au préalable ni avoir analysé leurs impacts sur l'activité quotidienne des médecins. Dans le cadre du présent article, les auteurs tentent de combler cette lacune. Au regard de l'impact des HTA sur le quotidien des médecins et de la controverse autour des HTA, en Allemagne par exemple, les auteurs invitent l'ASSM et la FMH à assumer leur rôle de forces régulatrices, et lors du processus d'introduction des HTA, de défendre les valeurs qui sont les leurs: l'éthique du médecin pour le FMH et la scientificité pour l'ASSM.

\section{Rückblick Managed Care}

Wir erinnern uns ebenfalls: Letzten Sommer wurde die Managed-Care-Vorlage vom Souverän mit 76\% Nein-Stimmen erstaunlich deutlich abgelehnt. Die FMH, anfangs für die Vorlage, schwenkte im letzten Moment um, was unleugbar einen wesentlichen Beitrag zumindest zur Deutlichkeit des Abstimmungsresultats geleistet hat. Die Gründe für diesen Meinungswechsel der FMH sind nicht zuletzt darin zu suchen, dass die Wirkung der anfangs als kostendämpfend beurteilten Anreize in Ärztenetzwerken mit Budgetmitverantwortung kritischer hinterfragt wurde. Wenn Arzt oder Netzwerk in einem BonusMalus-System bei Herauszögern und Vermeiden von Behandlungen und Weiterverweisungen eine mögliche Provision winkt und im gegenteiligen Fall eine Busse droht, dann muss das mit Blick auf die Behandlungsqualität und etwaige Mehrkosten durch teure, weil zu späte Behandlungen doch zumindest 


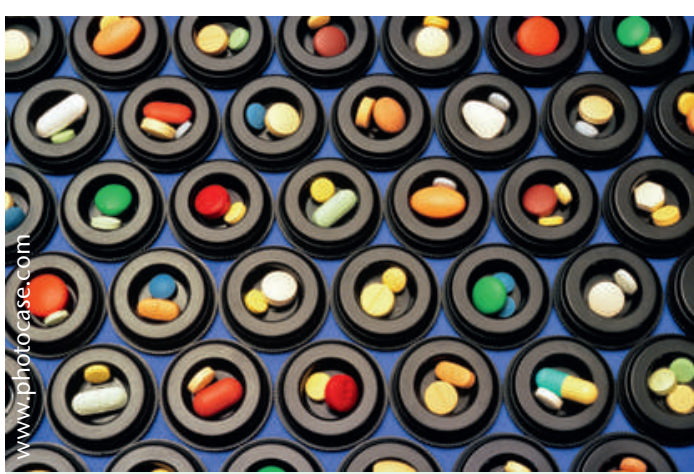

Health Technology Assessments sollen helfen, die Effizienz medizinischer Behandlungen zu beurteilen. Können sie das auch?

als problematisch bezeichnet werden. Und das hat starke Auswirkungen auf den Praxisalltag, was dem Zentralsekretariat der FMH offensichtlich erst sehr spät bewusst wurde.

\section{Ausblick HTA}

Zusammenfassend stellen wir fest: Von zwei hochgelobten Steuerungsmassnahmen zur Kostenreduktion im Gesundheitswesen ist die eine den Beweis für ihre Wirkung noch schuldig und die andere wurde vom Volk abgelehnt - nicht zuletzt, weil die FMH nach reiflicher Überlegung nicht mehr hinter ihr stehen konnte. Mit Health Technology Assessments (HTA) ist nun ein weiteres Steuerungsinstrument zur Kostenreduktion im Gesundheitswesen in den Startlöchern. HTAs sollen helfen, die Effizienz medizinischer Massnahmen zu messen, um zu objektivieren, welche von ihnen weiterhin bezahlt werden und welche nicht. Dieses Ansinnen ist an sich zu begrüssen, und die FMH engagiert sich denn auch für HTA, einerseits im Projekt SwissHTA (www.swisshta.ch), andererseits mit ihrer Mitgliedschaft im Swiss Medical Board SMB (www.medical-board.ch). Dass diese Zustimmung nicht ohne weiteres gegeben war, zeigt ein Beitrag von Daniel Herren, der später als Vertreter der FMH am Konsensus-Projekt von SwissHTA teilnahm, in dieser Zeitung [1]. Darin hielt er fest: «Aufgrund des einseitigen Diktats, vor allem der Interpharma, sowie der fehlenden Einflussmöglichkeit, vor allem in der Auswahl der Themen, der Experten und der Arbeitsweise, hat sich die FMH entschieden, vorerst nicht mitzumachen.» Diese Ausgangslage für die Einführung von HTA weckt vor oben Gesagtem nicht gerade Vertrauen. Doch HTA geniessen weiterhin die Unterstützung des Zentralsekretariats der FMH, ohne die FMH-Mitglieder diesbezüglich gebührend darüber informiert zu haben, was HTAs sind und wie sich deren Einführung auf den Arbeitsalltag von Ärztinnen und Ärzten auswirkt. Die Frage muss erlaubt sein: Bahnt sich hier ein ähnliches Fiasko an wie bei Managed Care, indem die FMH auch bezüglich HTA die Konsequenzen auf den Praxisalltag unterschätzt?

\section{Was sind HTAs?}

Es würde den Rahmen dieses Artikels sprengen, eingehend über HTA zu informieren. Der Kasten zeigt die wesentlichen Kritikpunkte - die nicht nur der VEMS einbringt, sondern namhafte Wissenschaftler. Zusammenfassend ist festzuhalten: Wir haben mit HTA eine weitere Steuerungsmassnahme mit einer Tendenz, den Indikations- und Behandlungsentscheid aus den Händen des Arztes zu nehmen und an fachfremde Gremien zu übergeben. Die Auswahl an Behandlungen wird insbesondere auch in der Grundversicherung verringert, der Arzt folglich in seiner Tätigkeit eingeschränkt - Konsequenzen also, über die das Zentralsekretariat der FMH seine Mitglieder doch zumindest adäquat informieren sollte. Grundsätzlich besteht hier das Dilemma, dass über HTA ohne QALYs wenig gewusst und noch viel geforscht werden muss, QALYs aber andererseits ausser in England und Kanada nicht verwendet werden oder, wie etwa in den USA, sogar gesetzlich verboten sind. Darüber keine basisdemokratische Diskussion zu führen, mit der Begründung, HTA seien die beste aller schlechten Lösungen, ist ein Fatalismus, den sich die Schweiz nicht leisten kann und muss.

\section{Blick nach Deutschland und Forderungen an die FMH}

In Deutschland ist das Institut für Qualität und Wirtschaftlichkeit im Gesundheitswesen IQWiG (www. iqwig.de) bezüglich HTA die federführende Kraft, und eine Suche auf Google nach dem Begriffspaar «Kritik» und «IQWiG» zeigt, dass nicht unbedingt das zugrunde gelegte Methodenpapier, jedoch dessen konkrete Arbeit weder als wissenschaftlich noch als unabhängig beurteilt wird, indem politisch gewollte Eingriffe auf statistisch nicht zulässige Art in das methodische Vorgehen erfolgen. Diese Kritik ist fundiert und kommt von durchaus kompetenten Stellen: Fachgesellschaften, Deutschem Ethikrat, Patientenschutzorganisationen und der Pharmazeutischen Industrie. Trotz aller Kontroversen um die Arbeit der Deutschen HTA-Behörde IQWiG ist doch festzuhalten, dass das IQWiG grundsätzlich bereit ist, seine Arbeit gemeinsam mit allen Akteuren des Gesundheitswesens zu leisten und für diese Arbeit eine Konsensusbasis zu finden. Das Papier «Allgemeine Methoden» des IQWiG wurde in harter Arbeit unter Einbezug dieser Akteure erfochten und ging im April dieses Jahres in die Überarbeitung zur Version 4.0, wozu auf der Website des IQWiG öffentlich um Eingaben gebeten wird. In der Schweiz hat ein solcher breit und öffentlich ausgetragener Konsensusprozess indes nie stattgefunden, vielmehr hat das Swiss Medical Board (SMB) sein Methodenpapier im Alleingang verfasst. Sollte uns dies nicht aufhorchen und kurz innehalten lassen? Oder machen SwissHTA und Swiss Medical Board ihre Arbeit tatsächlich so gut, dass kein Grund zur Sorge besteht? Der jüngste, auf www.vems.ch/ethical-board und in dieser Zeitung besprochene SMB-Bericht «Computertomogra- 


\section{Problemzonen rund um HTA}

\section{QALY}

Das Konzept der quality adjusted life years (QALY) ist umstritten, weil es alte, behinderte und polymorbide Patienten stigmatisiert. Ohne diese Grundlage sind HTAs aber schwer durchführbar [2].

Studiengrundlage

Die verwendeten Studien sind zwar evidenzbasiert. Ihre Verwendung ist aber oft statistisch problematisch, oder es werden Untergruppen gebildet, wodurch die Aussagekraft der Schlüsse wieder auf die Hypothesen-Stufe sinkt [3].

\section{Validierung}

Die Analyseresultate sind nicht prospektiv validiert und somit klinisch nicht überprüft, was zu schwer abschätzbaren Folgeschäden führen kann [4].

\section{Indikationsentscheid}

Der Indikationsentscheid wird tendenziell aus den Händen des Arztes genommen, womit man den Patienten um die eigentliche intellektuelle Leistung des Arztes bringt, die darin besteht, zusammen mit seinem Patienten zu entscheiden, welche Behandlung im individuellen Einzelfall zweckmässig ist [5].

\section{WZW, rechtliche Folgen}

Die Beschneidung der Hoheit über den Indikationsentscheid des Arztes hat direkte Implikationen auf die Handhabung der WZW-Kriterien (wirksam, wirtschaftlich, zweckmässig), seine Folgen (wirtschaftliche, medizinisch-ethische und rechtliche) hat aber der Arzt zu tragen [6].
phie-Scanner in der Abklärung der koronaren Herzerkrankung» legt eher das Gegenteil nahe (s. auch nachfolgenden Beitrag). Hier ist die gesamte FMH inklusive Basis gefordert, nicht nur der Zentralvorstand.

\section{Referenzen}

1 Herren D. HTA: How to assess? Schweiz Ärztezeitung. 2011;92(14):519.

2 www.ethikrat.org/dateien/pdf/stellungnahme-nutzenund-kosten-im-gesundheitswesen.pdf

3 http://dgepi.de/fileadmin/pdf/GEP_LL_english_f.pdf

4 Dies betrifft insbesondere Markov-Modelle mit Berechnungen von kurzen Beobachtungen, welche aus RCT gewonnen werden, aber bis an das Lebensende hochgerechnet werden, ohne dies mit Beobachtungsstudie abzusichern.

5 Im Einzelfall können durchaus alternative Pfade, die allenfalls aus Kostengründen durch den HTA-Prozess eliminiert werden, sinnvoll sein. Siehe etwa: www. deutsche-apotheker-zeitung.de/spektrum/ news/2013/05/24/fachgesellschaften-wollen-amverfahren-ruetteln/10155.html

6 https://hill.swisslex.ch/JournalPortal.mvc/ AssetDetail?assetGuid=a447f980-e0f0-448c-996fa95061200fb9 